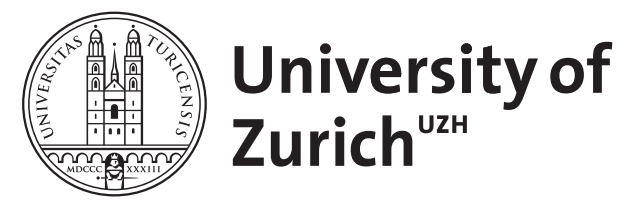

\title{
Klinik der Spastik - spastische Bewegungsstörung
}

Dietz, V

\begin{abstract}
Spastizität entwickelt sich als Folge einer Schädigung des Zentralnervensystems (ZNS). Klinisch ist die Spastik durch eine Muskelhypertonie und gesteigerte Eigenreflexe charakterisiert und mit unterschiedlichen Graden von Parese verbunden. Zusammen ergibt sich das Syndrom der spastischen Parese. Der Patient ist durch erschwerte und verlangsamte Bewegungsabläufe beeinträchtigt. Elektrophysiologische Untersuchungen funktioneller Arm- und Beinbewegungen (z.B. beim Gehen) zeigen eine verminderte Aktivierung der Arm- und Beinmuskeln. Dies erklärt sich durch den Ausfall aktivierender Signale motorischer Hirnzentren und funktioneller Reflexsysteme. Dieser Einfluss überwiegt die gesteigerte Eigenreflexaktivität. Die der Parese entsprechende verminderte Muskelaktivierung wird durch strukturelle Veränderungen der Muskelfasern teilweise kompensiert (z.B. Verlust von Sarkomeren). Deshalb kann durch eine antispastische Medikation, die eine Ausschaltung der Eigenreflexe zum Ziel hat, meist keine Funktionsverbesserung erreicht werden. Deren Einsatz ist aber bei immobilen Patienten sinnvoll. Bei mobilen Patienten wird durch ein funktionelles Training eine Funktionsverbesserung erreicht, die mit einem angepassten, d.h. verminderten spastischen Muskeltonus einhergeht
\end{abstract}

DOI: https://doi.org/10.1007/s00115-013-3933-2

Other titles: Clinical treatment of spasticity-spastic movement disorders

Posted at the Zurich Open Repository and Archive, University of Zurich ZORA URL: https://doi.org/10.5167/uzh-155876

Journal Article

Published Version

Originally published at:

Dietz, V (2013). Klinik der Spastik - spastische Bewegungsstörung. Der Nervenarzt, 84(12):1508-1511. DOI: https://doi.org/10.1007/s00115-013-3933-2 
Nervenarzt 2013 $\cdot 84: 1508-1511$

DOI 10.1007/s00115-013-3933-2

Online publiziert: 24. November 2013

(c) Springer-Verlag Berlin Heidelberg 2013

\section{Dietz}

Zentrum für Paraplegie, Universitätsklinik Balgrist, Zürich

\section{Klinik der Spastik - spastische Bewegungsstörung}

erhöhten Widerstand (• Abb. 2). Diese Beobachtungen führten früher (d. h. vor der Durchführung elektrophysiologischer Untersuchungen natürlicher Bewegungsabläufe) zu der Annahme, dass die gesteigerten Eigenreflexe zur spastischen Muskeltonuserhöhung führen und damit für die Bewegungsstörung verantwortlich sind. Dabei wurde nicht bedacht, dass bei natürlichen Bewegungsabläufen auch andere Reflexsysteme, wie die polysynaptischen (oder „Long-latency“-)Reflexe (die in der Klinik nicht getestet werden), wesentlich zur Bewegungsdurchführung beitragen [2].

\section{Spastische Bewegungsstörung}

Bei aktiven Bewegungen von Patienten mit spastischer Parese fehlt die bei Gesunden vorhandene Modulation der Muskelaktivität. Es kommt lediglich zu einer geringen tonischen Aktivierung der Extremitätenmuskeln. Insgesamt ist die Muskelaktivität sowohl bei Arm- [3] als auch Beinbewegungen ([4]; z. B. Gehen) im Vergleich zu Gesunden reduziert. Die

Der Beitrag entspricht einer aktualisierten Version der Veröffentlichung: Dietz V (2011) State of the Art - Spastik, PharmaFokus ZNS, 8:17-18.

\section{wegungsstörung ergeben. Diese ha- ben Konsequenzen für eine sachge- rechte Behandlung der Spastik.}

\section{Klinik der Spastik}

Die Diagnose einer spastischen Parese beruht auf der Untersuchung der Muskeleigenreflexe und des Muskeltonus beim sitzenden/liegenden, $d$. h. passiven Patienten. Nach einer akuten ZNS-Läsion (z. B. Schlaganfall) sind die Eigenreflexe auf der gelähmten Seite, als Folge einer verminderten präsynaptischen Hemmung der Ia-Fasern, schon früh gesteigert. Im weiteren Verlauf kommt es zu strukturellen Veränderungen der Muskelfasern (unter anderem Verlust von Sarkomeren [1]) und damit zur Entwicklung des spastischen Muskeltonus. Zusammen führen diese Veränderungen zum Bild der klinischen Spastik (• Abb. 1). Wird bei der klinischen Untersuchung ein passiver Extremitätenmuskel rasch gedehnt (Ashworth-Test), so kommt es im Gegensatz zum Gesunden zu einer tonischen Aktivierung des Muskels und damit zu einem

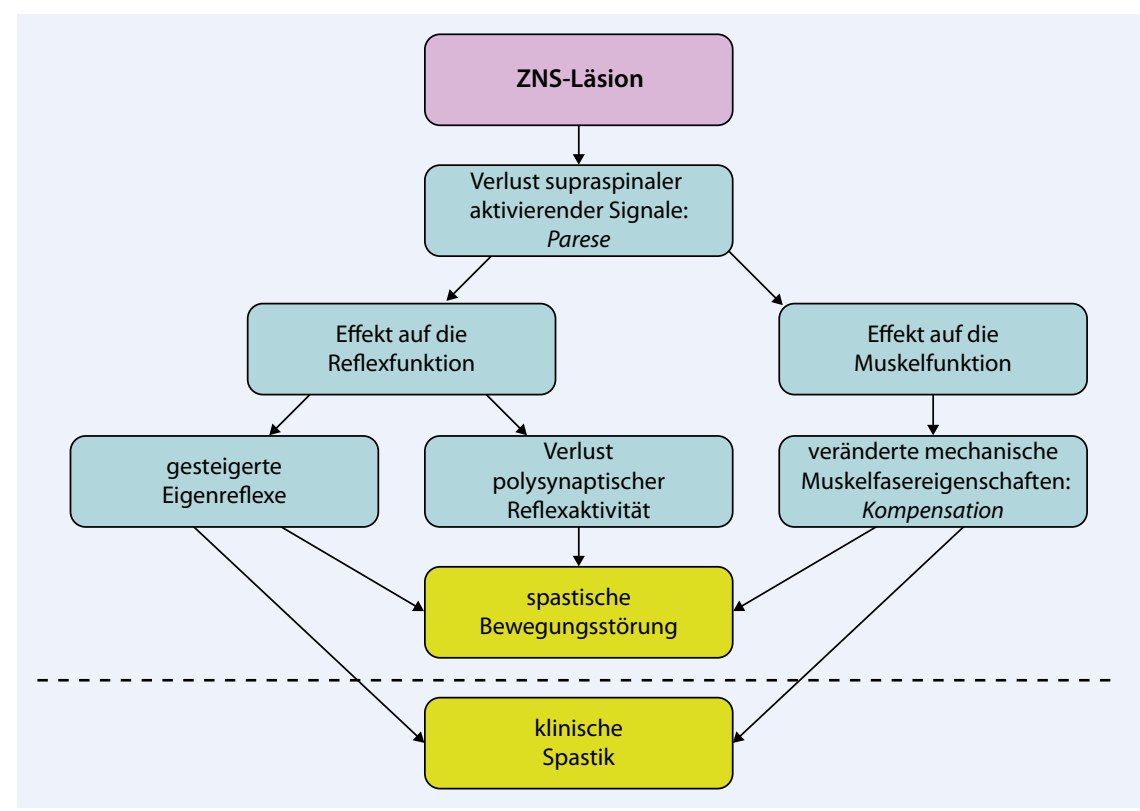

Abb. $1 \Delta$ Physiologische Mechanismen des spastischen Syndroms. Eine ZNS-Läsion führt zu einer veränderten Erregbarkeit spinaler Reflexe und einem Verlust aktivierender Signale motorischer Hirnzentren. Als Konsequenz kommt es zu strukturellen Veränderungen der Muskelfasern. In der Klinik führen gesteigerte Eigenreflexe und ein erhöhter Muskeltonus zur Diagnose der spastischen Parese. Bei der spastischen Bewegungsstörung kompensieren strukturelle Veränderungen der Muskelfasern teilweise die durch den Ausfall aktivierenden Impulse (von polysynaptischen Reflexen und motorischen Hirnzentren verursachte Parese; adaptiert nach [2]) 


\section{A Normaler Muskel}

\section{B Spastischer Muskel}
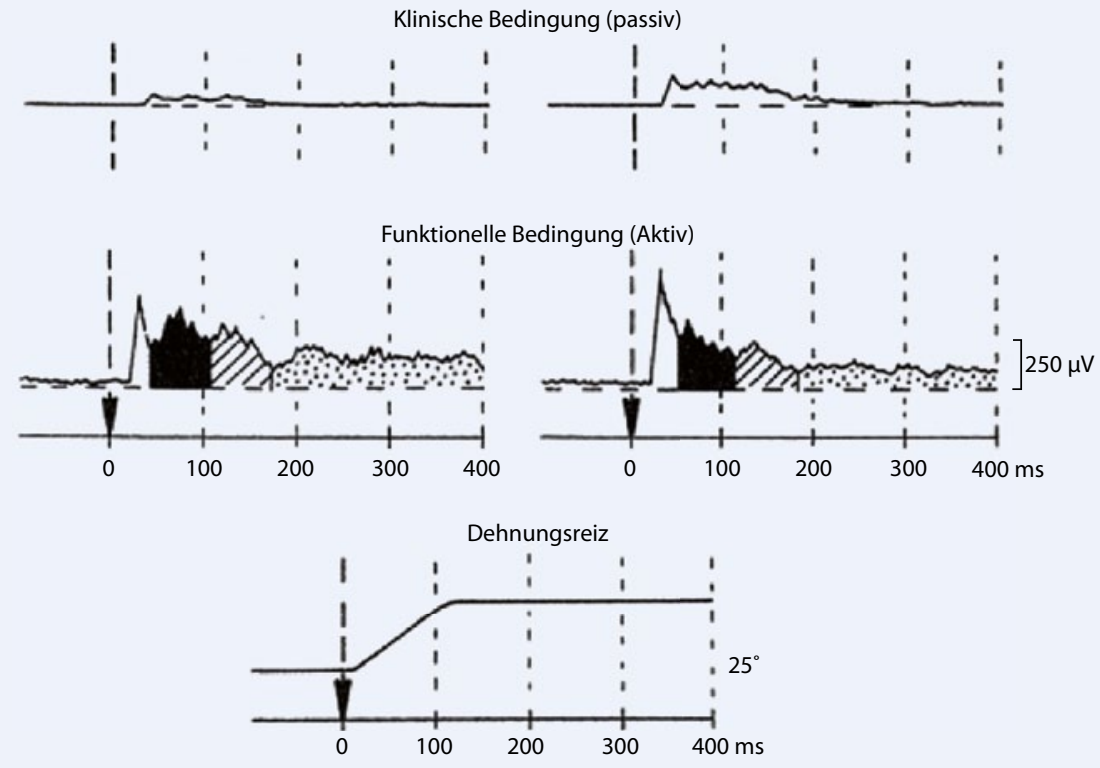

Abb. $2 \Delta$ Aktive und passive Bewegungsbedingung bei spastischer Parese. Die passive Bedingung (oben) entspricht der klinischen Untersuchung. Bei rascher Dehnung des M. brachioradialis kommt es im Gegensatz zu Gesunden bei der Spastik zu einer tonischen Muskelaktivierung und einem dadurch erhöhten Widerstand des gedehnten Muskels. Bei der aktiven Bewegungsbedingung (unten) ist die Aktivität des spastischen Muskels auf Dehnung, trotz der gesteigerten (Eigen-)Reflexe kurzer Latenz, im Vergleich zum normalen Muskel, vermindert. (Adaptiert nach [3])

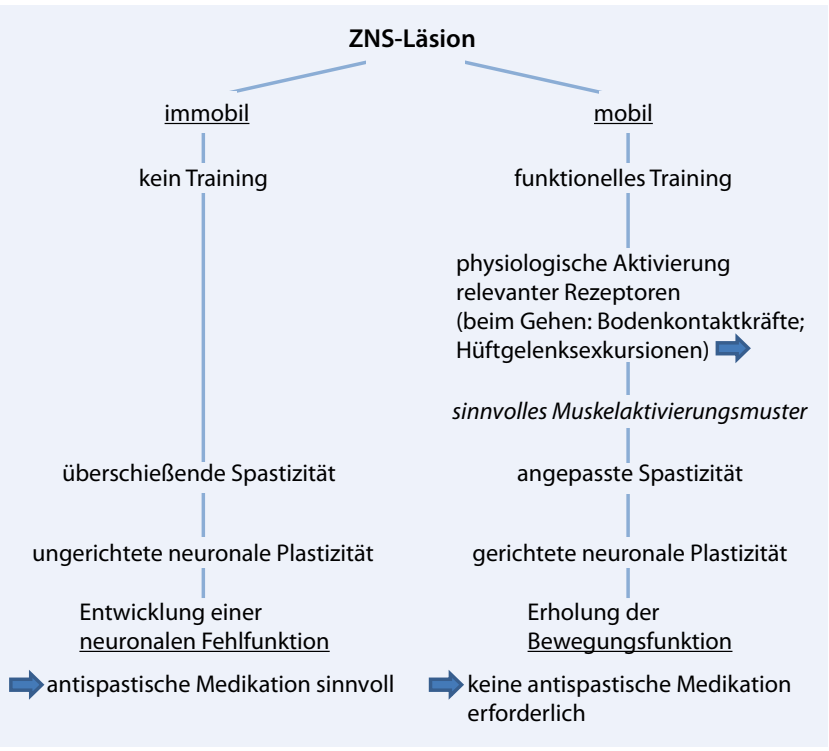

Abb. $3 \Delta$ Neuronale Funktionsveränderungen nach einer ZNS-Läsion. Es bestehen grundlegende Veränderungen der neuronalen Funktion nach einer ZNS-Schädigung (Schlaganfall, Querschnittlähmung), die von der Mobilität eines Patienten abhängen. Bei mobilen Patienten kommt es durch ein funktionelles Training, bei dem die Aktivierung physiologischer Rezeptoren ausreichend berücksichtigt wird, zu einer Funktionserholung als Folge einer gerichteten Neuroplastizität und Anpassung des spastischen Muskeltonus. Bei immobilen Patienten kommt es zu einem Überschießen des spastischen Muskeltonus und einer neuronalen Dysfunktion als Folge einer ungerichteten Neuroplastizität verminderte Muskelaktivierung ist durch den Ausfall erregender Signale motorischer Hirnzentren und auch durch die reduzierte Aktivität polysynaptischer Reflexe bei diesen Bewegungsabläufen der Arme (• Abb. 2) und Beine bedingt ([5], zur Übersicht s. [2]). Die gesteigerten Eigenreflexe spielen bei diesen natürlichen Bewegungsmustern weder bei Gesunden noch bei Patienten mit Spastik eine wesentliche Rolle [6]. Dagegen tragen die polysynaptischen Reflexe, die bei der klinischen Untersuchung nicht erfasst werden, bei Gesunden wesentlich zur Modulation der Aktivität besonders in den Antigravitationsmuskeln (Armbeuger und Fußstrecker) bei [2].

Diese Beobachtungen zeigen, dass die Tonusentwicklung bei natürlichen Bewegungsabläufen, wie dem Gehen, auf einem einfacheren Organisationsniveau als bei Gesunden erfolgt (zur Übersicht s. [6] und 1): Der für die Körperunterstützung in der Standphase des Gehens notwendige Muskeltonus erfolgt nicht wie bei Gesunden durch die modulierte Aktivierung der Streckermuskeln, sondern durch die Dehnung der gering tonisch aktivierten Beinmuskeln. Strukturelle Veränderungen des Muskels (z. B. Verlust von Sarkomeren), die in der Folge einer ZNS-Läsion stattfinden (beginnend 3 bis 5 Wochen nach Ereignis), können die Parese teilweise kompensieren und ermöglichen dadurch eine Körperunterstützung beim Gehen (• Abb. 1). Rasche Bewegungen können bei dieser einfachen Form der Regulation des Muskeltonus jedoch nicht mehr durchgeführt werden.

Zudem ist bei Patienten mit spastischer Hemiparese die neuronale Kopplung von Armen und Beinen als Grundlage der Gehfunktion [7] und der beider Arme bei kooperativen Handbewegungen [8] gestört. Nervenstimulation des nicht betroffenen Beins beim Gehen führt normalerweise zur beidseitigen Aktivierung der proximalen Armmuskeln, während die Stimulation des paretischen Beins weder zu einer Armmuskelantwort der betroffenen noch der nichtbetroffenen Seite führt [5]. Dies weist auf eine gestörte Kontrolle der Afferenz durch den kortikospinalen Trakt hin [9]. Diese Interaktion kann therapeutisch genutzt werden, indem durch Stimulation der nichtbetrof- 
fenen Seite auch die paretische Seite mitaktiviert wird.

Zusammengefasst ist die Muskelaktivierung bei Patienten mit Spastik im Vergleich zu Gesunden im passiven $\mathrm{Zu}$ stand, d. h. bei der klinischen Untersuchung, verstärkt, bei aktiven Bewegungen jedoch vermindert ( $\bullet$ Abb. 2). Die klinischen Befunde der Spastik können somit nicht auf die Bewegungsstörung übertragen werden.

Therapeutische Konsequenzen Die meisten antispastischen Medikamente sollen eine Reduktion des Muskeltonus durch Ausschaltung der über die Gruppe der Ia-Nervenfasern laufenden (Eigen-)Reflexe bewirken. Elektrophysiologische Untersuchungen natürlicher Bewegungsabläufe zeigen, dass ein spastischer Muskeltonus bei gehfähigen Patienten benötigt wird, um die Parese nach einer ZNSLäsion zu kompensieren [6]. Das heißt, bei mobilen Patienten können antispastische Medikamente die Parese verstärken und zu einer Funktionsverschlechterung führen. Bei diesen Patienten ist ein funktionelles Arm- und Bein-(Lokomotions-)Training indiziert. Dadurch kommt es zu einer gerichteten Neuroplastizität [10] mit verstärkter Arm-/Beinmuskelaktivierung ( $\bullet$ Abb. 3). Die Konsequenz ist eine Funktionsverbesserung, die mit einer verminderten Spastik einhergeht. Es wird weniger spastischer Muskeltonus benötigt, mit der Folge eines neuen Gleichgewichts zwischen Bewegungsfunktion und spastischem Muskeltonus [6]. Das heißt auch, dass ein erfolgreiches funktionelles Training einen gewissen spastischen Muskeltonus benötigt [11]. Rehabilitationsroboter können künftig eine wesentliche Rolle bei dieser funktionellen Therapie übernehmen [12].

Bei immobilen Patienten kommt es nach ca. einem Jahr zu einer neuronalen Dysfunktion [13, 14], die mit einer raschen Erschöpfbarkeit der Muskelaktivität einhergeht. Diese wird durch ein ungerichtetes Aussprossen von Rückenmarkfasern hervorgerufen [9]. Bei diesen Patienten können antispastische Medikamente (z. B. intrathekale Baclofen-Applikation) Spasmen lindern und die Pflege erleichtern.

Nervenarzt 2013 $\cdot 84: 1508-1511 \quad$ DOI 10.1007/s00115-013-3933-2

(c) Springer-Verlag Berlin Heidelberg 2013

V. Dietz

Klinik der Spastik - spastische Bewegungsstörung

\section{Zusammenfassung}

Spastizität entwickelt sich als Folge einer Schädigung des Zentralnervensystems (ZNS). Klinisch ist die Spastik durch eine Muskelhypertonie und gesteigerte Eigenreflexe charakterisiert und mit unterschiedlichen Graden von Parese verbunden. Zusammen ergibt sich das Syndrom der spastischen Parese. Der Patient ist durch erschwerte und verlangsamte Bewegungsabläufe beeinträchtigt. Elektrophysiologische Untersuchungen funktioneller Arm- und Beinbewegungen (z. B. beim Gehen) zeigen eine verminderte Aktivierung der Arm- und Beinmuskeln. Dies erklärt sich durch den Ausfall aktivierender Signale motorischer Hirnzentren und funktioneller Reflexsysteme. Dieser Einfluss überwiegt die gesteigerte Eigenreflexaktivität. Die der Pare- se entsprechende verminderte Muskelaktivierung wird durch strukturelle Veränderungen der Muskelfasern teilweise kompensiert (z. B. Verlust von Sarkomeren). Deshalb kann durch eine antispastische Medikation, die eine Ausschaltung der Eigenreflexe zum Ziel hat, meist keine Funktionsverbesserung erreicht werden. Deren Einsatz ist aber bei immobilen Patienten sinnvoll. Bei mobilen Patienten wird durch ein funktionelles Training eine Funktionsverbesserung erreicht, die mit einem angepassten, $d$. h. verminderten spastischen Muskeltonus einhergeht.

Schlüsselwörter

Spastizität · Zentralnervensystem ·

Muskelhypertonie · Parese $\cdot$ Reflexsysteme

\section{Clinical treatment of spasticity - spastic movement disorders}

\section{Summary}

Spasticity develops as a consequence of damage to the central nervous system (CNS). Clinically, spasticity is characterized by muscle hypertension and exaggerated reflexes and is associated with varying degrees of paresis. Together this results in the syndrome of spastic paresis. Patients suffer from impeded and retarded movement ability. Electrophysiological investigations of functional arm and leg movements (e.g. in walking) show a reduced activation of arm and leg muscles which can be explained by the loss of activating signals from motor brain centers and functional reflex systems. This effect predominates over the increased tendon-reflex activity. The reduced muscle activation caused by paresis is partially compensated by structural alterations of the muscle fibers (e.g. loss of sarcomeres). For this reason a functional improvement mostly cannot be achieved by antispastic medication which targets the deactivation of tendon-reflexes. However, they are useful in immobilized patients. In mobile patients functional improvement can be achieved by functional training which is accompanied by an adapted, i.e. reduced, spastic muscle tone.

\section{Keywords}

Spasticity $\cdot$ Central nervous system · Muscle hypertension · Paresis - Reflex system

\section{Korrespondenzadresse}

\section{Prof. Dr. V. Dietz}

Zentrum für Paraplegie, Universitätsklinik Balgrist

Forchstr. 340, 8008 Zürich

Schweiz

vdietz@paralab.balgrist.ch

\section{Einhaltung ethischer Richtlinien}

Interessenkonflikt. V. Dietz gibt an, dass kein Interessenkonflikt besteht. Alle im vorliegenden Manuskript beschriebenen Untersuchungen am Menschen wurden mit Zustimmung der zuständigen Ethik-Kommission, im Einklang mit nationalem Recht sowie ge- mäß der Deklaration von Helsinki von 1975 (in der aktuellen, überarbeiteten Fassung) durchgeführt. Von allen beteiligten Patienten liegt eine Einverständniserklärung vor.

\section{Literatur}

1. O'Dwyer NJ, Ada L (1996) Reflex hyperexcitability and muscle contracture in relation to spastic hypertonia. Curr Opin Neurol 9:451-455

2. Dietz V (2002) Proprioception and locomotor disorders. Nat Rev Neurosci 3:781-790

3. Ibrahim IK, Berger W, Dietz V (1993) Stretchinduced electromyographic activity and torque in spastic elbow muscles. Brain 116:971-989

4. Dietz V, Quintern J, Berger W (1981) Electrophysiological studies of gait in spasticity and rigidity. Brain 104:431-449 
5. Kloter E, Wirz M, Dietz V (2011) Locomotion in stroke subjects: interaction between unaffected and affected sides. Brain 134:721-731

6. Dietz V, Sinkjaer T (2007) Spastic movement disorder: impaired reflex function and altered muscle mechanics. Lancet Neurol 6:725-733

7. Dietz V (2002) Do human bipeds use quadrupedal coordination? Trends Neurosci 25:462-467

8. Dietz V, Macauda G, Schrafl-Altermatt M et al (2013) Neural coupling of cooperative hand movements: a reflex and fMRI study. Cereb Cortex (advance access DOI 10.1093/cercor/bkt285)

9. Lemon R (2008) Descending pathways in motor control. Annu Rev Neurosci 31:195-218

10. Beauparlant J, Brand R van den, Barraud $Q$ et al (2013) Undirected compensatory plasticity contributes to neuronal dysfunction after severe spinal cord injury. Brain 136:3347-3361

11. Dietz V, Fouad K (2013) Restoration of sensorimotor functions after spinal cord injury. Brain (advance access DOI 10.1093/brain/awt262)

12. Riener R, Lünenburger $L$, Maier IC et al (2010) Locomotor training in subjects with sensori-motor deficits: an overview of the robotic gait orthosis Lokomat. J Healthcare Engin 1:197-216

13. Dietz V, Müller R (2004) Degradation of neuronal function following a spinal cord injury: mechanisms and countermeasures. Brain 127:2221-2231

14. Dietz V, Grillner S, Trepp A et al (2009) Changes in spinal reflex and locomotor activity after complete spinal cord injury: a common mechanism? Brain 132:2196-2205

15. Dietz V (2011) State of the Art - Spastik, PharmaFokus ZNS. 8:17-18

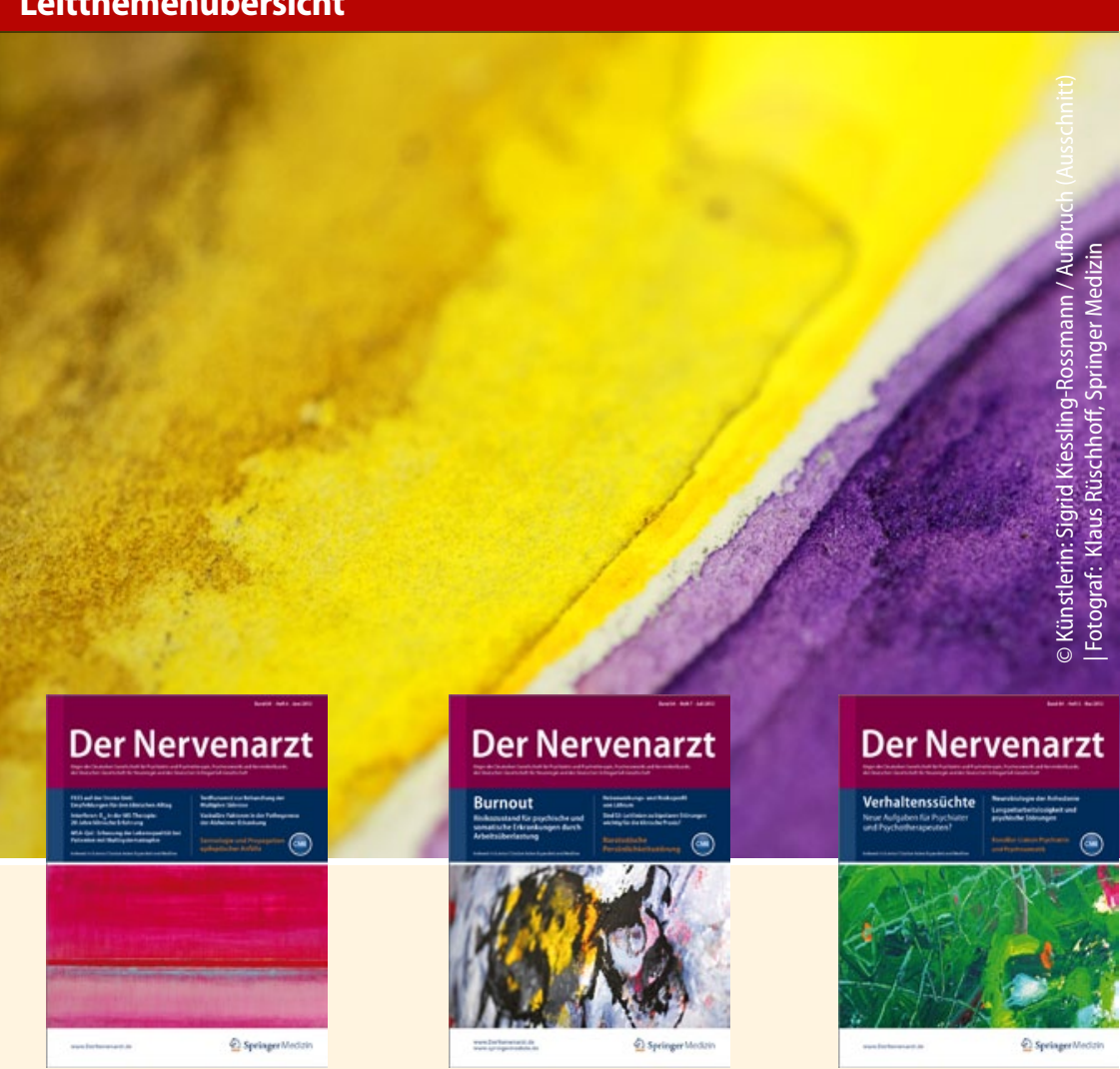

Der Nervenarzt bietet Ihnen jeden Monat umfassende und aktuelle Beiträge zu interessanten Themenschwerpunkten aus allen Bereichen der Neurologie und Psychiatrie. Möchten Sie ein bereits erschienenes Heft nachbestellen? Einzelne Ausgaben können Sie direkt bei unserem Kundenservice zum Preis von je EUR 36,- zzgl. Versandkosten beziehen:

2013

1/13 Kein Leitthema

2/13 Neurogenetik

3/13 Kein Leitthema

4/13 Immunvermittelte Enzephalomyelitiden

5/13 Verhaltenssüchte

6/13 Kein Leitthema

7/13 Burnout

8/13 Medizin in Bewegung

9/13 Psychiater im Nationalsozialismus Beispiele für Zivilcourage

10/13 Aktuelle Kontroversen der Neuroethik

11/13 Von der Therapie zur Prävention

12/13 Schmerz und Kopfschmerz

So erreichen Sie unseren Kundenservice:

Springer Customer Service Center GmbH Kundenservice Zeitschriften

Haberstr. 7

69126 Heidelberg

Tel.: +496221 345-4303

Fax: +49 $6221345-4229$

E-Mail: leserservice@springer.com

www.DerNervenarzt.de
2014

1/14 Schlaf

2/14 Tiefe Hirnstimulation in Neurologie und Psychiatrie

3/14 Forensik

4/14 Multimorbidität

5/14 Kein Leitthema

6/14 Bildgebung

7/14 Fahrerlaubnis bei psychiatrischen und neurologischen Erkrankungen

8/14 Prävention

9/14 Kein Leitthema

10/14 Prädiktion

11/14 Demographischer Wandel

12/14 Lifestyle

(Änderungen vorbehalten) 